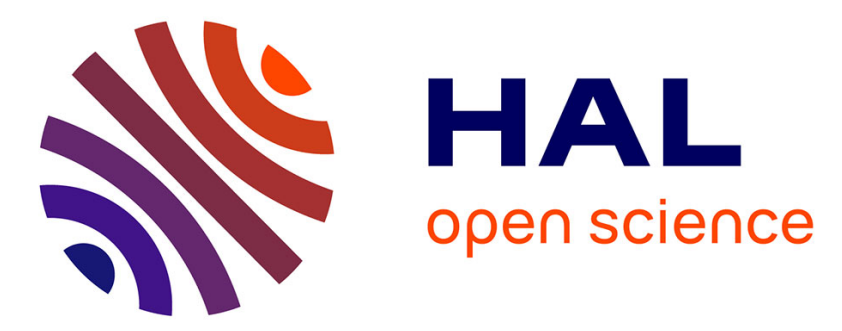

\title{
The unfitted HHO method for the Stokes problem on curved domains
}

\author{
Erik Burman, Guillaume Delay, Alexandre Ern
}

\section{To cite this version:}

Erik Burman, Guillaume Delay, Alexandre Ern. The unfitted HHO method for the Stokes problem on curved domains. ENUMATH - European Numerical Mathematics and Advanced Applications Conference 2019, Sep 2019, Egmond an Zee, Netherlands. hal-02438622

\section{HAL Id: hal-02438622 \\ https://hal.science/hal-02438622}

Submitted on 14 Jan 2020

HAL is a multi-disciplinary open access archive for the deposit and dissemination of scientific research documents, whether they are published or not. The documents may come from teaching and research institutions in France or abroad, or from public or private research centers.
L'archive ouverte pluridisciplinaire HAL, est destinée au dépôt et à la diffusion de documents scientifiques de niveau recherche, publiés ou non, émanant des établissements d'enseignement et de recherche français ou étrangers, des laboratoires publics ou privés. 


\title{
The unfitted HHO method for the Stokes problem on curved domains
}

\author{
Erik Burman* Guillaume Delay ${ }^{\dagger} \quad$ Alexandre Ern ${ }^{\ddagger}$
}

January 14, 2020

\begin{abstract}
We design a hybrid high-order (HHO) method to approximate the Stokes problem on curved domains using unfitted meshes. We prove inf-sup stability and a priori estimates with optimal convergence rates. Moreover, we provide numerical simulations that corroborate the theoretical convergence rates. A cell-agglomeration procedure is used to prevent the appearance of small cut cells.
\end{abstract}

\section{Introduction}

Generating meshes to solve problems posed on domains with a curved boundary can be a difficult task when high-order methods are used. The use of unfitted meshes that do not fit this boundary can circumvent this difficulty. In the framework of finite element methods, the main paradigm for unfitted methods [4] is the use of Nitsche's method [18] to enforce weakly the boundary conditions at the boundary. One difficulty with this method is the possible presence of small cut cells, i.e. cells that have only a small fraction of their volume inside the actual physical domain. These small cut cells can have an adverse effect on the conditioning of the system matrix and can even hamper convergence (see [9] for a recent study on the topic). The most common way to deal with the problem of small cut cells is to add a stabilizing term such as the ghost penalty [2]. In the present study, we use a cell-agglomeration technique to prevent the appearance of small cut cells $[15,3,20]$.

In order to easily handle the various shapes of the cells produced by the agglomeration process, we consider the hybrid high-order (HHO) method, which is a polyhedral method. HHO methods have been introduced recently in [11, 10]. As shown in [8], they are closely related to hybridizable discontinuous Galerkin methods and to nonconforming Virtual element methods. Moreover, the unfitted HHO method has already been studied in $[5,3]$ for elliptic interface problems. More precisely, it was adapted to the unfitted framework in [5], a mixed-order polynomial setting was considered with the cell unknowns being one degree higher than the face unknowns and a first algorithm for the cell-agglomeration procedure was provided. This study was continued in [3], where the use of a novel gradient reconstruction operator eliminated the

\footnotetext{
${ }^{*}$ Department of Mathematics, University College London, London, UK-WC1E 6BT, UK.

${ }^{\dagger}$ Sorbonne Université, CNRS, Université de Paris, Laboratoire Jacques-Louis Lions (LJLL), F-75005 Paris, France and Université Paris-Est, CERMICS (ENPC) and INRIA Paris, France.

${ }^{\ddagger}$ Université Paris-Est, CERMICS (ENPC), 77455 Marne-la-Vallée cedex 2, and INRIA Paris, France.
} 
requirement on the Nitsche's penalty parameter to be large enough. An improvement of the cell-agglomeration algorithm and numerical simulations were also provided.

The present study extends the unfitted HHO method presented in [3] to the Stokes problem. Unfitted schemes have already been used to approximate the Stokes problem in e.g. $[6,17,13,14]$. A HHO scheme was already provided on fitted meshes for the Stokes problem in [12] and for the Navier-Stokes equations in [1]. In addition to the usual stabilization and gradient reconstruction operators, a divergence reconstruction operator is also defined. We here focus on the Stokes problem in curved domains. The extension to the Stokes problem for two immiscible fluids separated by a curved interface will be treated in a future work. We also mention that a HDG scheme for the Stokes problem in curved domains was devised in [19].

Let $\Omega$ be a smooth domain in $\mathbb{R}^{d}, d \in\{2,3\}$ and $\Gamma=\partial \Omega$ its boundary. We consider the Stokes problem

$$
\begin{aligned}
& -\Delta \boldsymbol{u}+\nabla p=\boldsymbol{f} \quad \text { in } \Omega, \\
& \nabla \cdot \boldsymbol{u}=0 \quad \text { in } \Omega, \\
& \boldsymbol{u}=\boldsymbol{g} \quad \text { on } \Gamma \text {, }
\end{aligned}
$$

where $\boldsymbol{u}$ and $p$ are the velocity and pressure of the fluid. In the sequel, we denote $\boldsymbol{n}_{\Gamma}$ the unit outward normal of $\Omega$. For all $\boldsymbol{f} \in L^{2}\left(\Omega ; \mathbb{R}^{d}\right)$ and $\boldsymbol{g} \in H^{1 / 2}\left(\Gamma ; \mathbb{R}^{d}\right)$ with $\int_{\Gamma} \boldsymbol{g} \cdot \boldsymbol{n}_{\Gamma}=0$, the problem (1) admits a unique solution in $H^{1}\left(\Omega ; \mathbb{R}^{d}\right) \times L_{0}^{2}(\Omega)$, where $L_{0}^{2}(\Omega):=\left\{q \in L^{2}(\Omega) \mid \int_{\Omega} q=0\right\}$.

In Section 2, we introduce the unfitted HHO method for the Stokes problem on curved domains. We also state the main stability result in the form of an inf-sup condition and the main error estimates. In Section 3, we present some numerical simulations.

\section{The unfitted HHO method}

We consider a shape-regular polyhedral mesh sequence $\left(\mathcal{T}_{h}\right)_{h>0}$ in the sense of [10]. In particular, all the cells $T \in \mathcal{T}_{h}$ are assumed to have planar faces and straight edges. We denote $\rho>0$ the parameter that quantifies the regularity of the mesh. We denote $h_{T}$ the diameter of the cell $T \in \mathcal{T}_{h}$ and $\boldsymbol{n}_{T}$ its unit outward normal. We set conventionally $h:=\max _{T \in \mathcal{T}_{h}} h_{T}$. The meshes do not necessarily fit $\Omega$.

For all $T \in \mathcal{T}_{h}$, we denote $T^{\circ}:=T \cap \Omega,(\partial T)^{\circ}:=\partial T \cap \Omega$ and $T^{\Gamma}:=T \cap \Gamma$. Let $\mathbb{P}^{\ell}(S)$ (resp. $\left.\mathbb{P}^{\ell}\left(S ; \mathbb{R}^{d}\right), \mathbb{P}^{\ell}\left(S ; \mathbb{R}^{d \times d}\right)\right)$ be the space composed of scalar (resp. vectorial, matricial) polynomials of degree at most $\ell \geq 0$ in $S$. We denote $(\cdot, \cdot)_{S}$ the $L^{2}$-scalar product on $S$ and $\|\cdot\|_{S}$ the associated norm.

We assume that the meshes fulfill the following three assumptions.

Assumption 1 (Cut cells). There is $\delta \in(0,1)$ such that, for all $T \in \mathcal{T}_{h}$, there is $\widetilde{\boldsymbol{x}_{T}} \in T^{\circ}$ such that $B\left(\widetilde{\boldsymbol{x}_{T}}, \delta h_{T}\right) \subset T^{\circ}$.

Assumption 2 (Multiplicative trace inequality). There are $c_{\mathrm{mtr}}>0$ and $\theta_{\mathrm{mtr}} \geq 1$, such that for all $T \in \mathcal{T}_{h}$, there is $\check{\boldsymbol{x}}_{T} \in \mathbb{R}^{d}$ so that for all $\boldsymbol{v} \in H^{1}\left(T^{\dagger} ; \mathbb{R}^{d}\right)$ with $T^{\dagger}:=B\left(\check{\boldsymbol{x}}_{T} ; \theta_{\mathrm{mtr}} h_{T}\right),\|\boldsymbol{v}\|_{(\partial T)^{\circ}}+\|\boldsymbol{v}\|_{T^{\Gamma}} \leq c_{\mathrm{mtr}}\left(h_{T}^{-1 / 2}\|\boldsymbol{v}\|_{T^{\dagger}}+\|\boldsymbol{v}\|_{T^{\dagger}}^{1 / 2}\|\nabla \boldsymbol{v}\|_{T^{\dagger}}^{1 / 2}\right)$.

Assumption 3 (Resolving $T^{\dagger}$ ). There exists $N_{0} \in \mathbb{N}$ (independent from $h$ ) such that for every $T \in \mathcal{T}_{h}, T^{\dagger} \subset \Delta_{N_{0}}(T)$, where $\Delta_{0}(T):=T$ and $\Delta_{j+1}(T):=\left\{T^{\prime} \in\right.$ $\left.\mathcal{T}_{h} \mid \overline{T^{\prime}} \cap \overline{\Delta_{j}(T)} \neq \emptyset\right\}$ for all $j \geq 0$.

Assumption 1 means that there are no bad cut cells in the mesh. This assumption provides a discrete trace inequality [5], and it can be satisfied by the cell-agglomeration 
procedure described in [3] if the mesh is fine enough w.r.t. the curvature of $\Gamma$, see [5]. Assumption 2 is classical in the framework of unfitted finite element methods. It can be established if the mesh is fine enough w.r.t. the curvature of the boundary [5]. Assumption 3 is reasonable for meshes that are not too graded.

\subsection{The local discrete problem}

Let $k \geq 0$ be the face polynomial degree in the unfitted HHO method. The velocity is represented by a vector-valued polynomial of degree at most $k+1$ in every cell and a vector-valued polynomial of degree at most $k$ on every face. The pressure is represented by a polynomial of degree at most $k$ in every cell. The local degrees of freedom are denoted $\hat{\boldsymbol{u}}_{T}=\left(\boldsymbol{u}_{T}, \boldsymbol{u}_{\partial T}\right) \in \mathbb{P}^{k+1}\left(T^{\circ} ; \mathbb{R}^{d}\right) \times \mathbb{P}^{k}\left(\mathcal{F}_{(\partial T)^{\circ}} ; \mathbb{R}^{d}\right)=: \hat{\boldsymbol{U}}_{T}^{k}$ and $p_{T} \in \mathbb{P}^{k}\left(T^{\circ}\right)$, where $\mathbb{P}^{k}\left(\mathcal{F}_{(\partial T)^{\circ}} ; \mathbb{R}^{d}\right):=\prod_{F^{\circ} \in \mathcal{F}_{(\partial T)}} \mathbb{P}^{k}\left(F^{\circ} ; \mathbb{R}^{d}\right)$ and $\mathcal{F}_{(\partial T)^{\circ}}:=\left\{F^{\circ}:=\right.$ $\left.F \cap \Omega \mid F \in \mathcal{F}_{h}, F \subset \partial T\right\}$, with $\mathcal{F}_{h}$ the set of faces of $\mathcal{T}_{h}$.

We define the gradient reconstruction operator $\mathbb{G}_{T}^{k}: \hat{\boldsymbol{U}}_{T}^{k} \rightarrow \mathbb{P}^{k}\left(T^{\circ} ; \mathbb{R}^{d \times d}\right)$ such that for all $\hat{\boldsymbol{u}}_{T} \in \hat{\boldsymbol{U}}_{T}^{k}$ and all $\mathbb{q} \in \mathbb{P}^{k}\left(T^{\circ} ; \mathbb{R}^{d \times d}\right)$, we have

$$
\left(\mathbb{G}_{T}^{k}\left(\hat{\boldsymbol{u}}_{T}\right), \mathbb{q}\right)_{T^{\circ}}:=\left(\nabla \boldsymbol{u}_{T}, \mathbb{q}\right)_{T^{\circ}}+\left(\boldsymbol{u}_{\partial T}-\boldsymbol{u}_{T}, \mathbb{q} \boldsymbol{n}_{T}\right)_{(\partial T)^{\circ}}-\left(\boldsymbol{u}_{T}, \mathbb{q} \boldsymbol{n}_{\Gamma}\right)_{T^{\Gamma}} .
$$

In a similar way, we define the divergence reconstruction operator $D_{T}^{k}: \hat{\boldsymbol{U}}_{T}^{k} \rightarrow \mathbb{P}^{k}\left(T^{\circ}\right)$ such that for all $\hat{\boldsymbol{u}}_{T} \in \hat{\boldsymbol{U}}_{T}^{k}$ and all $q \in \mathbb{P}^{k}\left(T^{\circ}\right)$, we have

$$
\left(D_{T}^{k}\left(\hat{\boldsymbol{u}}_{T}\right), q\right)_{T^{\circ}}:=\left(\nabla \cdot \boldsymbol{u}_{T}, q\right)_{T^{\circ}}+\left(\boldsymbol{u}_{\partial T}-\boldsymbol{u}_{T}, q \boldsymbol{n}_{T}\right)_{(\partial T)^{\circ}}-\left(\boldsymbol{u}_{T}, q \boldsymbol{n}_{\Gamma}\right)_{T^{\Gamma}},
$$

so that $D_{T}^{k}\left(\hat{\boldsymbol{u}}_{T}\right)=\operatorname{Tr}\left(\mathbb{G}_{T}^{k}\left(\hat{\boldsymbol{u}}_{T}\right)\right)$. Furthermore, we define the stabilization operator

$$
s_{T}\left(\boldsymbol{u}_{T}, \boldsymbol{v}_{T}\right):=h_{T}^{-1}\left(\Pi_{(\partial T)^{\circ}}^{k}\left(\boldsymbol{u}_{\partial T}-\boldsymbol{u}_{T}\right), \boldsymbol{v}_{\partial T}-\boldsymbol{v}_{T}\right)_{(\partial T)^{\circ}}+h_{T}^{-1}\left(\boldsymbol{u}_{T}, \boldsymbol{v}_{T}\right)_{T^{\Gamma}},
$$

where $\Pi_{(\partial T)^{\circ}}^{k}$ denotes the $L^{2}$-orthogonal projection onto $\mathbb{P}^{k}\left(\mathcal{F}_{(\partial T)^{\circ}} ; \mathbb{R}^{d}\right)$. We then define the following local bilinear and linear forms such that for all $\hat{\boldsymbol{v}}_{T}, \hat{\boldsymbol{w}}_{T} \in \hat{\boldsymbol{U}}_{T}^{k}$ and all $q_{T} \in \mathbb{P}^{k}\left(T^{\circ}\right)$,

$$
\begin{aligned}
a_{T}\left(\hat{\boldsymbol{v}}_{T}, \hat{\boldsymbol{w}}_{T}\right) & :=\left(\mathbb{G}_{T}^{k}\left(\hat{\boldsymbol{v}}_{T}\right), \mathbb{G}_{T}^{k}\left(\hat{\boldsymbol{w}}_{T}\right)\right)_{T^{\circ}}+s_{T}\left(\hat{\boldsymbol{v}}_{T}, \hat{\boldsymbol{w}}_{T}\right), \\
b_{T}\left(\hat{\boldsymbol{v}}_{T}, q_{T}\right) & :=\left(D_{T}^{k}\left(\hat{\boldsymbol{v}}_{T}\right), q_{T}\right)_{T^{\circ}}, \\
\ell_{T}^{a}\left(\hat{\boldsymbol{w}}_{T}\right) & :=\left(\boldsymbol{f}, \boldsymbol{w}_{T}\right)_{T^{\circ}}+\left(\boldsymbol{g}, h_{T}^{-1} \boldsymbol{w}_{T}-\mathbb{G}_{T}^{k}\left(\hat{\boldsymbol{w}}_{T}\right) \boldsymbol{n}_{\Gamma}\right)_{T^{\Gamma}}, \\
\ell_{T}^{b}\left(q_{T}\right) & :=-\left(\boldsymbol{g}, q_{T} \boldsymbol{n}_{\Gamma}\right)_{T^{\Gamma}} .
\end{aligned}
$$

Note that $s_{T}$ and $\mathbb{G}_{T}^{k}\left(\hat{\boldsymbol{u}}_{T}\right)$ are similar to the operators proposed in [3].

Remark 2.1. (Variants) The gradient reconstruction operators can also be defined in $\nabla \mathbb{P}^{k+1}\left(T^{\circ} ; \mathbb{R}^{d}\right)$ instead of $\mathbb{P}^{k}\left(T^{\circ} ; \mathbb{R}^{d \times d}\right)$ (see for instance [5]). Moreover one can also use cell unknowns in $\mathbb{P}^{k}\left(T^{\circ} ; \mathbb{R}^{d}\right)$ (instead of $\mathbb{P}^{k+1}\left(T^{\circ} ; \mathbb{R}^{d}\right)$ ) away from the interface provided the stabilization operator from $[10]$ is used.

\subsection{The global discrete problem}

The global unknowns are $\hat{\boldsymbol{u}}_{h} \in \mathbb{P}^{k+1}\left(\mathcal{T}_{h} ; \mathbb{R}^{d}\right) \times \mathbb{P}^{k}\left(\mathcal{F}_{h} ; \mathbb{R}^{d}\right)=: \hat{\boldsymbol{U}}_{h}^{k}$ and $p_{h} \in \mathbb{P}^{k}\left(\mathcal{T}_{h}\right)=$ : $P_{h}^{k}$. For all $T \in \mathcal{T}_{h}, \hat{\boldsymbol{u}}_{T}$ and $p_{T}$ are the local components of $\hat{\boldsymbol{u}}_{h}$ and $p_{h}$ attached to $T$ (see Section 2.1). Let $P_{h *}^{k}:=\left\{q_{h} \in P_{h}^{k} \mid \int_{\Omega} q_{h}=0\right\}$. We define the global bilinear forms $a_{h}\left(\hat{\boldsymbol{v}}_{h}, \hat{\boldsymbol{w}}_{h}\right):=\sum_{T \in \mathcal{T}_{h}} a_{T}\left(\hat{\boldsymbol{v}}_{T}, \hat{\boldsymbol{w}}_{T}\right), b_{h}\left(\hat{\boldsymbol{v}}_{h}, q_{h}\right):=\sum_{T \in \mathcal{T}_{h}} b_{T}\left(\hat{\boldsymbol{v}}_{T}, q_{T}\right)$, and the 
global linear forms $\ell_{h}^{a}\left(\hat{\boldsymbol{w}}_{h}\right):=\sum_{T \in \mathcal{T}_{h}} \ell_{T}^{a}\left(\hat{\boldsymbol{v}}_{T}\right), \ell_{h}^{b}\left(q_{h}\right):=\sum_{T \in \mathcal{T}_{h}} \ell_{T}^{b}\left(q_{T}\right)$. The discrete global problem reads: find $\left(\hat{\boldsymbol{u}}_{h}, p_{h}\right) \in Y_{h}^{k}:=\hat{\boldsymbol{U}}_{h}^{k} \times P_{h *}^{k}$ such that

$$
\begin{aligned}
a_{h}\left(\hat{\boldsymbol{u}}_{h}, \hat{\boldsymbol{v}}_{h}\right)-b_{h}\left(\hat{\boldsymbol{v}}_{h}, p_{h}\right) & =\ell_{h}^{a}\left(\hat{\boldsymbol{v}}_{h}\right), \\
b_{h}\left(\hat{\boldsymbol{u}}_{h}, q_{h}\right) & =\ell_{h}^{b}\left(q_{h}\right),
\end{aligned}
$$

for all $\left(\hat{\boldsymbol{v}}_{h}, q_{h}\right) \in Y_{h}^{k}$. This discrete global problem can be solved in an efficient way by means of a static condensation procedure as described e.g. in [7, 12]. Specifically, the global problem that actually has to be solved involves only the face degrees of freedom of the velocity and the mean pressure value in every cell. The other degrees of freedom can be computed in a post-processing step by means of local solves.

\subsection{Stability and error estimates}

For all $\hat{\boldsymbol{v}}_{h} \in \hat{\boldsymbol{U}}_{h}^{k}$, we denote $\left\|\hat{\boldsymbol{v}}_{h}\right\|_{*}^{2}:=\sum_{T \in \mathcal{T}_{h}}\left\|\nabla \boldsymbol{v}_{T}\right\|_{T^{\circ}}^{2}+h_{T}^{-1}\left\|\boldsymbol{v}_{\partial T}-\boldsymbol{v}_{T}\right\|_{(\partial T)^{\circ}}^{2}+$ $h_{T}^{-1}\left\|\boldsymbol{v}_{T}\right\|_{T^{\Gamma}}^{2}$ and for all $\left(\hat{\boldsymbol{v}}_{h}, q_{h}\right) \in Y_{h}^{k}$, we denote $\left\|\left(\hat{\boldsymbol{v}}_{h}, q_{h}\right)\right\|_{\#}^{2}:=\left\|\hat{\boldsymbol{v}}_{h}\right\|_{*}^{2}+\left\|q_{h}\right\|_{\Omega^{2}}^{2}$. We also denote $A_{h}\left(\left(\hat{\boldsymbol{v}}_{h}, q_{h}\right),\left(\hat{\boldsymbol{w}}_{h}, r_{h}\right)\right):=a_{h}\left(\hat{\boldsymbol{v}}_{h}, \hat{\boldsymbol{w}}_{h}\right)-b_{h}\left(\hat{\boldsymbol{w}}_{h}, q_{h}\right)+b_{h}\left(\hat{\boldsymbol{v}}_{h}, r_{h}\right)$. A numerical analysis leads to the following results that we state here without proof.

Theorem 4 (Inf-sup condition). Under the assumption 1, there exists $\beta>0$, depending only on $k, \delta$ and $\rho$, such that for every $\left(\hat{\boldsymbol{v}}_{h}, q_{h}\right) \in Y_{h}^{k}$, we have

$$
\beta\left\|\left(\hat{\boldsymbol{v}}_{h}, q_{h}\right)\right\|_{\#} \leq \sup _{\left(\hat{\boldsymbol{w}}_{h}, r_{h}\right) \in Y_{h}^{k}} \frac{A_{h}\left(\left(\hat{\boldsymbol{v}}_{h}, q_{h}\right),\left(\hat{\boldsymbol{w}}_{h}, r_{h}\right)\right)}{\left\|\left(\hat{\boldsymbol{w}}_{h}, r_{h}\right)\right\|_{\#}} .
$$

Moreover, there exists a unique solution $\left(\hat{\boldsymbol{u}}_{h}, p_{h}\right) \in Y_{h}^{k}$ to (6).

Sketch of the proof. - We prove the coercivity and the continuity of $a_{h}$ w.r.t. the norm $\|\cdot\|_{*}$ by proceeding as in [3].

- Let $\left(\hat{\boldsymbol{v}}_{h}, q_{h}\right) \in Y_{h}^{k}$. Using the test function $\left(\hat{\boldsymbol{w}}_{h}, r_{h}\right):=\left(\hat{\boldsymbol{v}}_{h}, q_{h}\right)$ proves $\left\|\hat{\boldsymbol{v}}_{h}\right\|_{*}^{2} \leq$ $C S\left\|\left(\hat{\boldsymbol{v}}_{h}, q_{h}\right)\right\|_{\#}$, where $S:=\sup _{\left(\hat{\boldsymbol{w}}_{h}, r_{h}\right) \in Y_{h}^{k}} \frac{A_{h}\left(\left(\hat{\boldsymbol{v}}_{h}, q_{h}\right),\left(\hat{\boldsymbol{w}}_{h}, r_{h}\right)\right)}{\left\|\left(\hat{\boldsymbol{w}}_{h}, r_{h}\right)\right\|_{\#}}$ and $C$ has the same dependencies as $\beta$.

- We use the surjectivity of the $\nabla$. operator to prove that $\left\|q_{h}\right\|_{\Omega}^{2} \leq C\left(S^{2}+\left\|\hat{\boldsymbol{v}}_{h}\right\|_{*}^{2}+\right.$ $\left.\sum_{T \in \mathcal{T}_{h}} h_{T}^{2}\left\|\nabla q_{T}\right\|_{T^{\circ}}^{2}+\sum_{F \in \mathcal{F}_{h}} h_{F}\left\|\left[q_{T}\right]_{F}\right\|_{F^{\circ}}^{2}\right)$.

- Using the test function $\left(\hat{\boldsymbol{w}}_{h}, r_{h}\right):=\left(\hat{\boldsymbol{w}}_{h}, 0\right)$ where $\hat{\boldsymbol{w}}_{T}:=\left(-h_{T}^{2} \nabla q_{T}, 0\right)$ proves $\sum_{T \in \mathcal{T}_{h}} h_{T}^{2}\left\|\nabla q_{T}\right\|_{T^{\circ}}^{2} \leq C\left(S^{2}+\left\|\hat{\boldsymbol{v}}_{h}\right\|_{*}^{2}\right)$.

- Using the test function $\left(\hat{\boldsymbol{w}}_{h}, q_{h}\right):=\left(\hat{\boldsymbol{w}}_{h}, 0\right)$ with $\hat{\boldsymbol{w}}_{T}:=\left(0, \boldsymbol{w}_{\partial T}\right)$ where $\boldsymbol{w}_{\partial T \mid F}:=$ $h_{F}\left[q_{T}\right]_{F} \boldsymbol{n}_{F}$ for all $T \in \mathcal{T}_{h}$, proves $\sum_{F \in \mathcal{F}_{h}} h_{F}\left\|\left[q_{T}\right]_{F}\right\|_{F^{\circ}}^{2} \leq C\left(S^{2}+\left\|\hat{\boldsymbol{v}}_{h}\right\|_{*}^{2}\right)$.

- This shows the inf-sup condition and thus the well-posedness of (6).

Theorem 5 (Error estimates). Under the assumptions 1, 2 and 3, there exsits $C>0$, depending on $k, \delta, c_{\mathrm{mtr}}, N_{0}$ and $\rho$, such that, if $(u, p)$ belongs to $H^{k+2}(\Omega) \times H^{k+1}(\Omega)$, we have

$$
\left(\sum_{T \in \mathcal{T}_{h}}\left\|\nabla u-\nabla u_{T}\right\|_{T^{\circ}}^{2}+\left\|p-p_{T}\right\|_{T^{\circ}}^{2}\right)^{1 / 2} \leq C h^{k+1}\left(|u|_{H^{k+2}(\Omega)}+|p|_{H^{k+1}(\Omega)}\right) .
$$

Remark 2.2. Contrary to the classical Nitsche's method, we do not need here any parameter to be large enough. This is a consequence of the construction of $\mathbb{G}_{T}^{k}\left(\hat{\boldsymbol{u}}_{T}\right)$ in (2). For more details, the reader can refer to [3] and [16] for similar ideas using FEM. 


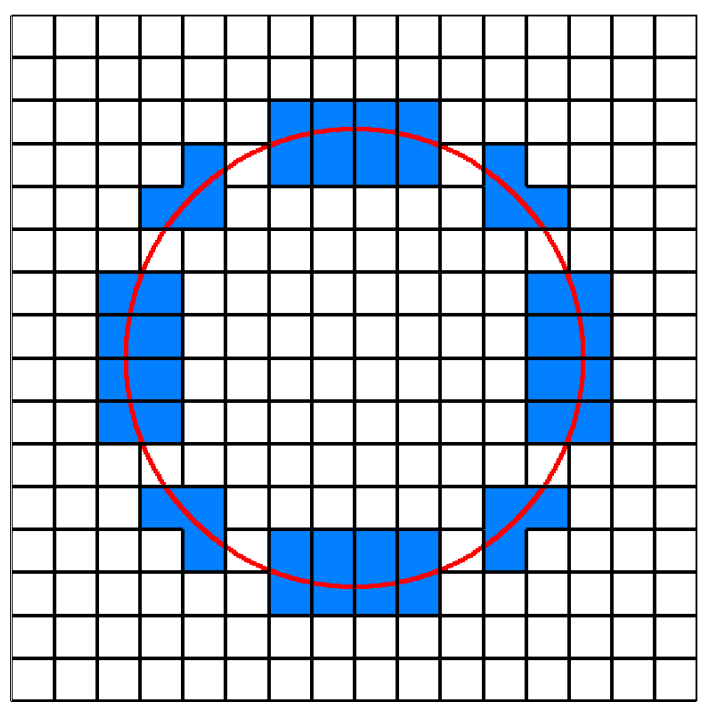

Figure 1: The mesh and the computational domain for $h=1 / 16$. The highlighted cells are the ones that are agglomerated. The boundary of the domain is in red.

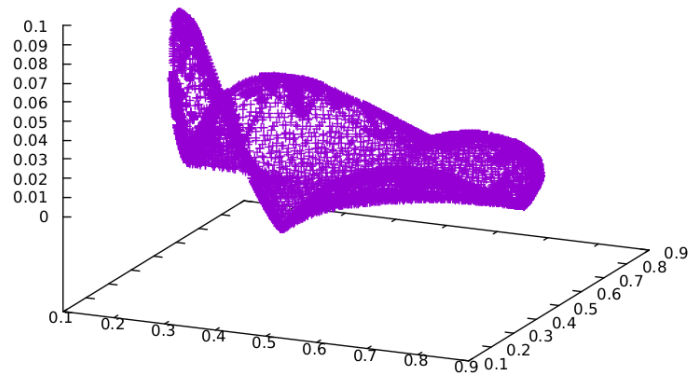

Figure 2: Euclidean velocity norm $(h=1 / 16, k=1)$.

\section{Numerical simulations}

On the circular domain $\Omega:=C((0.5,0.5) ; 1 / 3)$ (see Fig. 1), we consider the exact solution $u_{1}(x, y):=X^{2}\left(X^{2}-2 X+1\right) Y\left(4 Y^{2}-6 Y+2\right), u_{2}(x, y):=-Y^{2}\left(Y^{2}-2 Y+\right.$ 1) $X\left(4 X^{2}-6 X+2\right)$, and $p(x, y):=\sin (X+Y)$ where $X:=x-0.5, Y:=y-0.5$. The circular domain $\Omega$ is embedded into the unit square $(0,1)^{2}$ which is meshed with a uniform Cartesian mesh. In a pre-processing step, we use the cell-agglomeration technique described in [3]. Static condensation is used to decrease the total number of degrees of freedom. The global problem is solved by means of a LU decomposition. The numerical developments follow the DiSk ++ framework [7] and are available in the proton ${ }^{1}$ library. The profiles of the Euclidean velocity norm and the pressure are shown in Figures 2 and 3, respectively.

The cells lying completely outside the domain are not considered. The results of the numerical simulations are reported in Table 1. The rates of convergence are computed by comparing the result of one refinement step to the previous one. We recover the convergence rates stated in Theorem 5 .

\footnotetext{
${ }^{1}$ https://github.com/wareHHOuse
} 


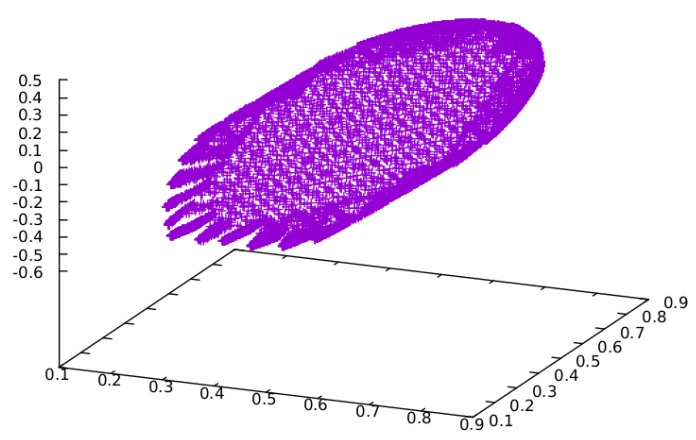

Figure 3: Pressure field $(h=1 / 16, k=1)$.

Table 1: Convergence of the errors for various polynomial orders

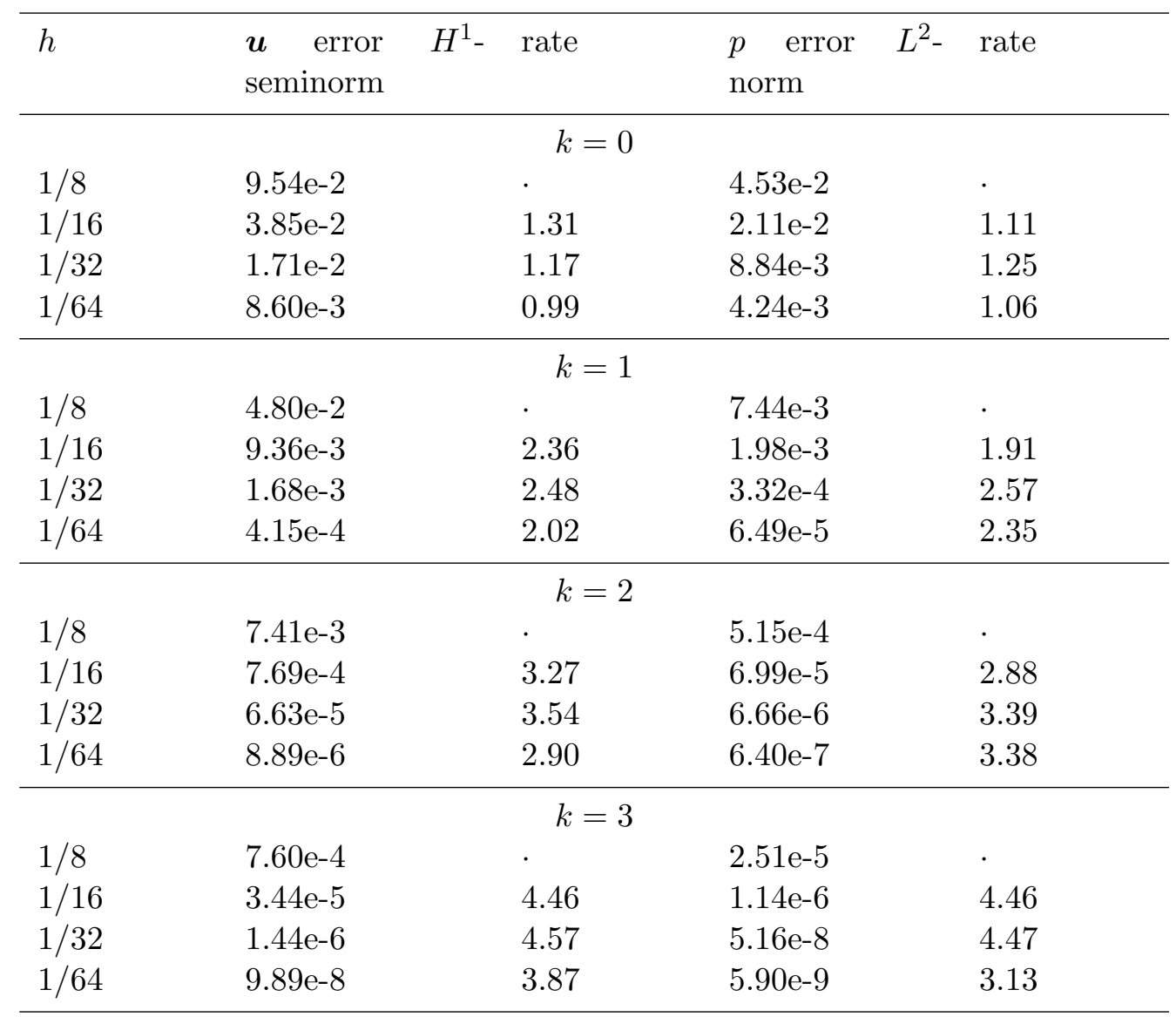

Note that, as in [3], in every cut cell, the interface is represented by $2^{r}$ segments, and every cut cell is then triangulated for the purpose of numerical integration. In the computations in Table 1, we used $r=11$. We think that the slightly lower rate of convergence observed for the pressure for $k=3$ and $h=1 / 64$ is due to a geometrical error that diminishes when $r$ is increased (at the price of a more expensive assembly of the system matrix). 


\section{References}

[1] Botti, L., Di Pietro, D. A. Droniou, J.: A hybrid high-order method for the incompressible Navier-Stokes equations based on Temam's device. J. Comput. Phys. 376, 786-816 (2019)

[2] Burman, E.: Ghost penalty. C. R. Math. Acad. Sci. Paris. 348(21-22), $1217-$ $1220(2010)$

[3] Burman, E., Cicuttin, M., Delay, G., Ern, A.: An unfitted Hybrid HighOrder method with cell agglomeration for elliptic interface problems. submitted. https://hal.archives-ouvertes.fr/hal-02280426/

[4] Burman, E., Claus, S., Hansbo, P., Larson, M. G., Massing, A.: CutFEM: discretizing geometry and partial differential equations. Internat. J. Numer. Methods Engrg. 104(7), 472-501 (2015)

[5] Burman, E., Ern., A.: An unfitted hybrid high-order method for elliptic interface problems. SIAM J. Numer. Anal. 56(3), 1525-1546 (2018)

[6] Burman, E., Hansbo, P.: Fictitious domain methods using cut elements: III. A stabilized Nitsche method for Stokes' problem. ESAIM Math. Model. Anal. 48(3), 859-874 (2014)

[7] Cicuttin, M., Di Pietro, D. A., Ern, A.: Implementation of discontinuous skeletal methods on arbitrary-dimensional, polytopal meshes using generic programming. J. Comput. Appl. Math. 344, 852-874 (2018)

[8] Cockburn, B., Di Pietro, D. A., Ern, A.: Bridging the Hybrid High-Order and Hybridizable Discontinuous Galerkin methods. ESAIM: Math. Model Numer. Anal. (M2AN) 50(3), 635-650 (2016)

[9] de Prenter, F., Lehrenfeld, C., Massing, A.: A note on the stability parameter in Nitsche's method for unfitted boundary value problems. Comput. Math. Appl. 75(12), 4322-4336 (2018).

[10] Di Pietro, D. A., Ern, A.: A Hybrid High-Order locking-free method for linear elasticity on general meshes. Comput. Meth. Appl. Mech. Engrg. 283, 1-21 (2015)

[11] Di Pietro, D. A., Ern, A., Lemaire, S.: An arbitrary-order and compact- stencil discretization of diffusion on general meshes based on local reconstruction operators. Comput. Meth. Appl. Math. 14(4), 461-472 (2014)

[12] Di Pietro, D. A., Ern, A., Linke , A., Schieweck, F.: A discontinuous skeletal method for the viscosity-dependent Stokes problem. Comput. Methods Appl. Mech. Engrg. 306, 175-195 (2016)

[13] Fournié, M., Lozinski, A.: Stability and optimal convergence of unfitted extended finite element methods with Lagrange multipliers for the Stokes equations. In: Lect. Notes Comput. Sci. Eng., pp. 143-182, Springer, Cham (2017)

[14] Guzmán, J., Olshanskii, M.: Inf-sup stability of geometrically unfitted Stokes finite elements. Math. Comp. 87(313), 2091-2112 (2018) 
[15] Johansson, A., Larson, M. G.: A high order discontinuous Galerkin Nitsche method for elliptic problems with fictitious boundary. Numer. Math. 123(4), 607-628 (2013)

[16] Lehrenfeld, C.: Removing the stabilization parameter in fitted and unfitted symmetric Nitsche formulations. arXiv:1603.00617 (2016)

[17] Massing, A., Larson, M. G., Logg, A., Rognes, M. E.: A stabilized Nitsche fictitious domain method for the Stokes problem. J. Sci. Comput. 61(3), 604$628(2014)$

[18] Nitsche., J.: Über ein Variationsprinzip zur Lösung von Dirichlet-Problemen bei Verwendung von Teilräumen, die keinen Randbedingungen unterworfen sind. Abh. Math. Sem. Univ. Hamburg 36, 9-15 (1971)

[19] Solano, M., Vargas, F.: A high order HDG method for Stokes flow in curved domains. J. Sci. Comput.79(3), 1505-1533 (2019)

[20] Sollie, W. E. H., Bokhove, O., van der Vegt, J. J. W.: Space-time discontinuous Galerkin finite element method for two-fluid flows. J. Comput. Phys. 230(3), 789-817 (2011) 